\title{
Effects of Hall Current on Unsteady MHD Couette Flow of Class-II in a Rotating System
}

\author{
G. S. Seth ${ }^{\dagger}$ and J. K. Singh \\ Department of Applied Mathematics, Indian School of Mines, Dhanbad (Jharkhand) -826004, India \\ †Corresponding Author Email: gsseth_ism@yahoo.com \\ (Received May 9, 2012; accepted February 10, 2013)
}

\begin{abstract}
Unsteady hydromagnetic Couette flow of class-II of a viscous incompressible electrically conducting fluid in a rotating system with Hall effects in the presence of a uniform transverse magnetic field is studied. Both the fluid and plates of the channel are assumed to be at rest when time $t^{\prime} \leq 0$ and fluid flow within the channel is induced due to non-torsional oscillations of the upper plate in its own plane with a velocity $U\left(t^{\prime}\right)$ about a non-zero uniform velocity $U_{0}$ at time $t^{\prime}>0$. Exact solution of the governing equations is obtained by Laplace transform technique. Asymptotic behavior of the solution is analyzed for small and large values of rotation parameter $K^{2}$ and magnetic parameter $M^{2}$ when time $t>>1$. The numerical values of the fluid velocity are depicted graphically whereas that of shear stress at the plates are presented in tabular form for various values of Hall current parameter $m$, rotation parameter $K^{2}$, magnetic parameter $M^{2}$ and frequency parameter $\omega$.
\end{abstract}

Keywords: MHD Couette flow of class-II, Rotation, Oscillations, Stokes-Ekman boundary layers, Modified Ekman boundary layers, Hartmann boundary layer.

\section{NOMENCLATURE}

$\begin{array}{ll}B_{0} & \text { uniform transverse magnetic field } \\ K^{2} & \text { rotation parameter } \\ L & \text { width of the channel } \\ m & \text { Hall current parameter } \\ M^{2} & \text { magnetic parameter } \\ p & \text { Laplace transform parameter } \\ p^{*} & \text { modified pressure including centrifugal } \\ & \text { force } \\ t & \text { non-dimensional time } \\ U_{0} & \text { uniform mean velocity in } x \text {-direction }\end{array}$

\section{INTRODUCTION}

Investigation of unsteady hydromagnetic flow of a viscous, incompressible and electrically conducting fluid assumes significance because transient nature of fluid flow may be expected at the start-up time of so many MHD devices viz. MHD energy generators, MHD pumps, induced type pumps used in nuclear reactors, MHD accelerators, MHD flow meters etc.. Keeping in view this fact unsteady MHD Couette flow

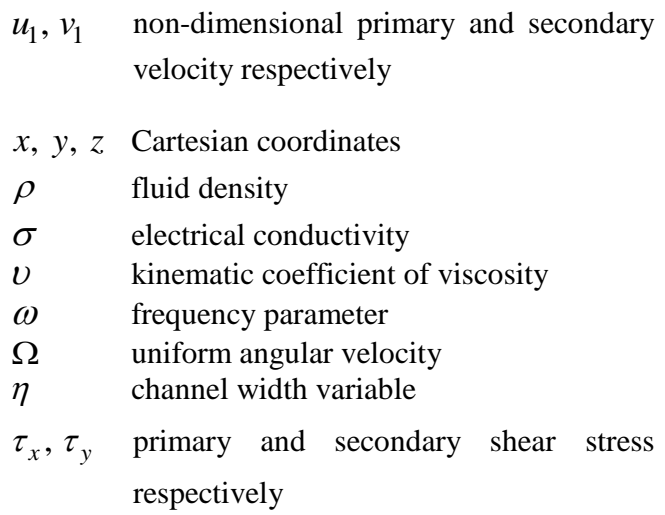

of a viscous, incompressible and electrically conducting fluid is investigated by a number of researchers. Mentioned may be made of the research studies of Tao (1960), Katagiri (1962), Muhuri (1963), Soundalgekar (1967), Mishra and Muduli (1980), Singh and Kumar (1983) and Seth et al. (2011a). Unsteady hydromagnetic Couette flow in a rotating system finds widespread applications in geophysics, planetary sciences and also in many areas of industrial engineering. In such types of 
flow Coriolis and magnetic forces play an important role in determining flow features of the problem. It may be noted that Coriolis force is much stronger than the inertial and viscous forces and it is comparable in magnitude with magnetic force. Keeping in view importance of such fluid flow problems Seth et al. (1982, 1988, 2009, 2010a, 2010b, 2011b, 2012), Chandran et al. (1993), Singh et al. (1994), Singh (2000), Ghosh and Pop (2004), Hayat et al. (2004a, 2004b, 2004c), Das et al. (2009), Guria et al. (2009) and Beg et al. (2011) investigated unsteady MHD Couette flow of a viscous, incompressible and electrically conducting fluid in a rotating system considering different aspects of the problem. On the basis of the above mentioned research studies on MHD Couette flow we are of opinion that MHD Couette flow may be induced in two ways and it can be put into two classes, namely, (i) MHD Couette flow of class-I and (ii) MHD Couette flow of class-II. The fluid flow which is induced due to the movement of a plate, when fluid is bounded by a stationary plate placed at a finite distance from the moving plate, may be recognized as MHD Couette flow of class-I. This fluid flow is similar to the fluid flow induced by a moving plate when free stream is stationary. The fluid flow past a stationary plate which is induced due to movement of a plate placed at a finite distance from the stationary plate may identified as MHD Couette flow of class-II. This fluid flow is similar to the flow past a stationary plate due to moving free stream. Research studies carried out by Seth et al. (1982, 1988, 2010a, 2010b, 2011b, 2012), Chandran et al. (1993), Singh et al. (1994), Ghosh (2002), Ghosh and Pop (2004), Guria et al. (2008, 2009), Das et al. (2009) and Beg et al. (2011) belong to MHD Couette flow of class-I whereas research investigations of Singh (2000), Hayat et al. (2004a, 2004b, 2004c), Seth et al. (2009, 2011c), Seth and Singh (2011) belong to MHD Couette flow of class-II.

It is well known that in an ionized fluid, where density is very low and/or the magnetic field is strong, the effects of Hall current become significant as mentioned by Cowling (1957) because Hall current induces secondary flow in the flow-field. Taking into account this fact Ghosh and Pop (2004) and Seth et al. (2012) studied Hall effects on unsteady MHD Couette flow in a rotating environment which belongs to MHD Couette flow of class-I whereas Hayat et al. (2004c) and Seth et al. (2009) investigated effects of Hall current on oscillatory MHD Couette flow of class-II in a rotating system considering different aspects of the problem.

Present investigation deals with the study of unsteady MHD Couette flow of class-II of a viscous, incompressible and electrically conducting fluid in a rotating system with Hall effects in the presence of a uniform transverse magnetic field applied parallel to the axis of rotation. Both the fluid and plates of the channel are assumed to be at rest when time $t^{\prime} \leq 0$ and fluid flow within the channel is induced due to non-torsional oscillations of the upper plate in its own plane with a velocity $U\left(t^{\prime}\right)$ about a non-zero uniform velocity $U_{0}$ at time $t^{\prime}>0$. Such study assumes importance because both Hall current and rotation induce secondary flow (i.e. cross flow) in the flow-field.

\section{MATHEMATICAL FORMULATION AND SOLUTION}

Consider flow of a viscous, incompressible and electrically conducting fluid between two infinite parallel plates $z=0$ and $z=L$ in the presence of a uniform transverse magnetic field $B_{0}$ which is applied parallel to $z$-axis. The fluid and channel rotate in counter clockwise direction with a uniform angular velocity $\Omega$ about $z$-axis. Both the fluid and plates of the channel are assumed to be at rest at time $t^{\prime} \leq 0$. Upper plate $z=L$ starts executing non-torsional oscillations in its own plane with a velocity $U\left(t^{\prime}\right)$ about a non-zero uniform mean velocity $U_{0}$ in $x$ direction at time $t^{\prime}>0$ and the lower plate $z=0$, which coincides with $x y$-plane, is kept fixed. Geometry of the problem is presented in Fig.1. It is assumed that the induced magnetic field produced by fluid motion is neglected in comparison to applied one. This is justified because magnetic Reynolds number is very small for metallic liquid and partially ionized fluids (Cramer and Pai, 1973). Also no applied or polarization voltage exists i.e. electric field $\vec{E} \equiv 0$. This corresponds to the case when no energy is added or extracted from the fluid by electrical means (Meyer, 1958). Since plates are of infinite extent in $x$ and $y$-directions, all physical quantities except pressure depend on $z$ and $t^{\prime}$ only.

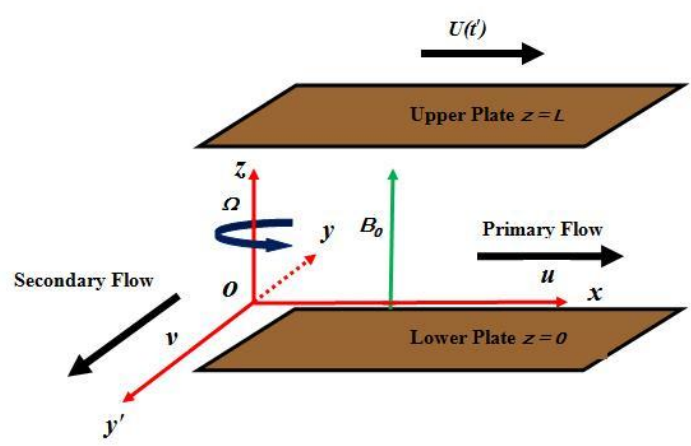

Fig. 1. Geometry of the problem

In view of the assumptions made above the governing equations for the fluid flow problem in a rotating system with Hall effects are given by

$$
\begin{aligned}
& \frac{\partial u}{\partial t^{\prime}}-2 \Omega v \\
& =-\frac{1}{\rho} \frac{\partial p^{*}}{\partial x}+v \frac{\partial^{2} u}{\partial z^{2}}-\frac{\sigma B_{0}^{2}}{\rho\left(1+m^{2}\right)}(u-m v) \\
& \frac{\partial v}{\partial t^{\prime}}+2 \Omega u \\
& =-\frac{1}{\rho} \frac{\partial p^{*}}{\partial y}+v \frac{\partial^{2} v}{\partial z^{2}}-\frac{\sigma B_{0}^{2}}{\rho\left(1+m^{2}\right)}(v+m u) \\
& 0=-\frac{1}{\rho} \frac{\partial p^{*}}{\partial z}
\end{aligned}
$$


where $u, v, v, \sigma, \rho, m=\omega_{e} \tau_{e}, \omega_{e}, \tau_{e}$ and $p^{*}$ are, respectively, fluid velocity in $\mathrm{x}$-direction, fluid velocity in y-direction, kinematic coefficient of viscosity, electrical conductivity of the fluid, fluid density, Hall current parameter, cyclotron frequency, electron collision time and modified pressure including centrifugal force.

The initial and boundary conditions are given by

$u=v=0$ for $\quad 0 \leq z \leq L \quad$ and $t^{\prime} \leq 0$

$u=v=0 \quad$ at $z=0 \quad$ for $t^{\prime}>0$

$u=U\left(t^{\prime}\right), v=0 \quad$ at $z=L \quad$ for $t^{\prime}>0$

Equation (3) shows that modified pressure $p^{*}$ is uniform along $z$-axis i.e. axis of rotation. Taking into the consideration of research studies made on MHD Couette flow till now, we are of the opinion that MHD Couette flow may be classified in two forms, namely, (i) MHD Couette flow of class-I and (ii) MHD Couette flow of class-II. The fluid flow induced due to movement of a plate, when fluid is bounded by a stationary plate placed at a finite distance from the moving plate, may be identified as MHD Couette flow of class-I. This fluid flow is similar to the flow induced due to movement of a plate when free stream is stationary. The fluid flow past a stationary plate, which is induced due to movement of a plate placed at a finite distance from the stationary plate, may be regarded as MHD Couette flow of class-II. This fluid flow is similar to the flow past a stationary plate due to moving free stream. For unsteady MHD Couette flow of class-I the pressure gradient terms $-\frac{1}{\rho} \frac{\partial p^{*}}{\partial x}$ and $-\frac{1}{\rho} \frac{\partial p^{*}}{\partial y}$, which are present in Eqs. (1) and (2) respectively, are not considered by Seth et al (1982, 1988, 2010a, 2010b, 2011b, 2012), Chandran et al. (1993), Singh et al. (1994), Ghosh (2002), Ghosh and Pop (2004), Guria et al. (2008, 2009), Das et al. (2009) and Beg et al. (2011). This assumption is justified and it is clearly evident from Eq. (5). For unsteady MHD Couette flow of class-II values of pressure gradient terms in Eqs. (1) and (2) are obtained with the help of boundary conditions, Eq. (6) which are given below

$$
\begin{aligned}
& -\frac{1}{\rho} \frac{\partial p^{*}}{\partial x}=\frac{\partial U}{\partial t^{\prime}}+\frac{\sigma B_{0}{ }^{2} U}{\rho\left(1+m^{2}\right)}, \\
& -\frac{1}{\rho} \frac{\partial p^{*}}{\partial y}=2 U \Omega+\frac{\sigma m B_{0}{ }^{2} U}{\rho\left(1+m^{2}\right)}
\end{aligned}
$$

Making use of Eq. (7), the Eqs. (1) and (2) reduce to

$$
\begin{aligned}
& \frac{\partial u}{\partial t^{\prime}}-2 \Omega v \\
& =\frac{\partial U}{\partial t^{\prime}}+v \frac{\partial^{2} u}{\partial z^{2}}-\frac{\sigma B_{0}^{2}}{\rho\left(1+m^{2}\right)}[(u-U)-m v]
\end{aligned}
$$

$$
\begin{aligned}
& \frac{\partial v}{\partial t^{\prime}}+2 \Omega(u-U) \\
& =v \frac{\partial^{2} v}{\partial z^{2}}-\frac{\sigma B_{0}^{2}}{\rho\left(1+m^{2}\right)}[v+m(u-U)]
\end{aligned}
$$

Equations (8) and (9), in non-dimensional form, become

$$
\begin{aligned}
& \frac{\partial u_{1}}{\partial t}-2 K^{2} v_{1} \\
& =\frac{\partial F}{\partial t}+\frac{\partial^{2} u_{1}}{\partial \eta^{2}}-\frac{M^{2}}{1+m^{2}}\left[\left(u_{1}-F\right)-m v_{1}\right]
\end{aligned}
$$

$\frac{\partial v_{1}}{\partial t}+2 K^{2}\left(u_{1}-F\right)$

$=\frac{\partial^{2} v_{1}}{\partial \eta^{2}}-\frac{M^{2}}{1+m^{2}}\left[v_{1}+m\left(u_{1}-F\right)\right]$

where $\eta=z / L, u_{1}=u / U_{0}, v_{1}=v / U_{0}, U=U_{0} F(t)$, $M^{2}=L^{2} B_{0}{ }^{2}(\sigma / \rho v)$ is magnetic parameter which is square of Hartmann number and $K^{2}=\Omega L^{2} / v$ is rotation parameter which is reciprocal of Ekman number.

The initial and boundary conditions, Eq. (4) to Eq. (6), in non-dimensional form, become

$$
\begin{array}{lll}
u_{1}=v_{1}=0 & \text { for } 0 \leq \eta \leq 1 & \text { and } t \leq 0 \\
u_{1}=v_{1}=0 & \text { at } \eta=0 & \text { for } t>0 \\
u_{1}=F(t), v_{1}=0 & \text { at } \eta=1 & \text { for } t>0
\end{array}
$$

Combining Eq. (10) with Eq. (11), we obtain

$$
\begin{aligned}
& \frac{\partial q}{\partial t}+i\left[\frac{m M^{2}}{1+m^{2}}+2 K^{2}\right](q-F) \\
& =\frac{\partial F}{\partial t}+\frac{\partial^{2} q}{\partial \eta^{2}}-\frac{M^{2}}{1+m^{2}}(q-F)
\end{aligned}
$$

where $q=u_{1}+i v_{1}$.

The initial and boundary conditions, Eq. (12) to Eq. (14), in compact form, become

$$
\begin{array}{lll}
q=0 & \text { for } 0 \leq \eta \leq 1 & \text { and } t \leq 0 \\
q=0 & \text { at } \eta=0 & \text { for } t>0 \\
q=F(t) & \text { at } \eta=1 & \text { for } t>0
\end{array}
$$

Since fluid flow is of oscillatory nature we may assume $F(t)$ in the following form

$$
F(t)=1+\left(a e^{i \omega t}+b e^{-i \omega t}\right)
$$


where $a$ and $b$ are complex constants and $b$ is complex conjugate of $a . \omega=\omega^{\prime} L^{2} / v$ is frequency parameter where $\omega^{\prime}$ is frequency of oscillations.

Using Laplace transform, Eq. (15) subject to the initial condition, Eq. (16) reduces to

$$
\begin{aligned}
& \frac{d^{2} \bar{q}}{d \eta^{2}}-\left[p+\frac{M^{2}}{1+m^{2}}+i\left(\frac{m M^{2}}{1+m^{2}}+2 K^{2}\right)\right] \bar{q} \\
& =-\left[p+\frac{M^{2}}{1+m^{2}}+i\left(\frac{m M^{2}}{1+m^{2}}+2 K^{2}\right)\right] \bar{F}
\end{aligned}
$$

where $\bar{q}=\int_{0}^{\infty} e^{-p t} q(\eta, t) d t, \bar{F}=\int_{0}^{\infty} e^{-p t} F(t) d t$ and $p$ being Laplace transform parameter.

Boundary conditions, Eq. (17) and Eq. (18) with use of Eq. (19) after taking Laplace transform become

$\bar{q}=0 \quad$ at $\eta=0$

$$
\bar{q}=\bar{F}(p)=\frac{1}{p}+\frac{a}{p-i \omega}+\frac{b}{p+i \omega} \quad \text { at } \eta=1
$$

Solution of Eq. (20) subject to the boundary conditions, Eq. (21) and Eq. (22) is given by

$$
\begin{aligned}
\bar{q} & =\left(\frac{1}{p}+\frac{a}{p-i \omega}+\frac{b}{p+i \omega}\right) \\
& \times\left(1-\frac{\sinh \lambda(1-\eta)}{\sinh \lambda}\right)
\end{aligned}
$$

where $\lambda=\left[p+M^{2}+i N^{2}\right]^{1 / 2}$ and $N^{2}=\frac{m M^{2}}{1+m^{2}}+2 K^{2}$.

Taking inverse Laplace transform of Eq. (23), we obtain

$$
\begin{aligned}
& q(\eta, t) \\
& =\left[1-\frac{\sinh \left(\alpha_{1}+i \beta_{1}\right)(1-\eta)}{\sinh \left(\alpha_{1}+i \beta_{1}\right)}\right] \\
& +\left[1-\frac{\sinh \left(\alpha_{2}+i \beta_{2}\right)(1-\eta)}{\sinh \left(\alpha_{2}+i \beta_{2}\right)}\right] a e^{i \omega t} \\
& +\left[1-\frac{\sinh \left(\alpha_{3} \pm i \beta_{3}\right)(1-\eta)}{\sinh \left(\alpha_{3} \pm i \beta_{3}\right)}\right] b e^{-i \omega t} \\
& -\sum_{n=1}^{\infty} 2 n \pi \sin n \pi \eta\left(\frac{1}{s_{1}}+\frac{a}{s_{1}-i \omega}+\frac{b}{s_{1}+i \omega}\right) e^{s_{1} t}
\end{aligned}
$$

for $\omega \neq \mathrm{N}^{2}$

where

$$
\alpha_{1}=\frac{1}{\sqrt{2}}\left[\left\{\frac{M^{4}}{\left(1+m^{2}\right)^{2}}+N^{4}\right\}^{1 / 2}+\frac{M^{2}}{1+m^{2}}\right]^{1 / 2},
$$

$$
\begin{aligned}
& \beta_{1}=\frac{1}{\sqrt{2}}\left[\left\{\frac{M^{4}}{\left(1+m^{2}\right)^{2}}+N^{4}\right\}^{1 / 2}-\frac{M^{2}}{1+m^{2}}\right]^{1 / 2}, \\
& \alpha_{2}=\frac{1}{\sqrt{2}}\left[\left\{\frac{M^{4}}{\left(1+m^{2}\right)^{2}}+\left(\omega+N^{2}\right)^{2}\right\}^{1 / 2}+\frac{M^{2}}{1+m^{2}}\right]^{1 / 2}, \\
& \beta_{2}=\frac{1}{\sqrt{2}}\left[\left\{\frac{M^{4}}{\left(1+m^{2}\right)^{2}}+\left(\omega+N^{2}\right)^{2}\right\}^{1 / 2}-\frac{M^{2}}{1+m^{2}}\right]^{1 / 2}, \\
& \alpha_{3}=\frac{1}{\sqrt{2}}\left[\left\{\frac{M^{4}}{\left(1+m^{2}\right)^{2}}+\left(\omega-N^{2}\right)^{2}\right\}^{1 / 2}+\frac{M^{2}}{1+m^{2}}\right]^{1 / 2}, \\
& \beta_{3}=\frac{1}{\sqrt{2}}\left[\left\{\frac{M^{4}}{\left(1+m^{2}\right)^{2}}+\left(\omega-N^{2}\right)^{2}\right\}^{1 / 2}-\frac{M^{2}}{1+m^{2}}\right]^{1 / 2}, \\
& \text { and } s_{1}=-\left[n^{2} \pi^{2}+\frac{M^{2}}{1+m^{2}}+i N^{2}\right]^{2}
\end{aligned}
$$

The upper and lower signs in Eq. (24) are considered for $\omega<N^{2}$ and $\omega>N^{2}$ respectively.

When $\omega=N^{2}$ i.e. when the natural frequency $N^{2}$, which is due to rotation and Hall current, is equal to the impressed frequency $\omega$ the inverse Laplace transform of Eq. (23) gives

$$
\begin{aligned}
& q(\eta, t) \\
& =\left[1-\frac{\sinh \left(\alpha_{1}^{\prime}+i \beta_{1}{ }^{\prime}\right)(1-\eta)}{\sinh \left(\alpha_{1}{ }^{\prime}+i \beta_{1}{ }^{\prime}\right)}\right] \\
& +\left[1-\frac{\sinh \left(\alpha_{2}{ }^{\prime}+i \beta_{2}{ }^{\prime}\right)(1-\eta)}{\sinh \left(\alpha_{2}{ }^{\prime}+i \beta_{2}{ }^{\prime}\right)}\right] a e^{i \omega t} \\
& +\left[1-\frac{\sinh \alpha_{3}{ }^{\prime}(1-\eta)}{\sinh \alpha_{3}{ }^{\prime}}\right] b e^{-i \omega t} \\
& -\sum_{n=1}^{\infty} 2 n \pi \sin n \pi \eta\left(\frac{1}{s_{1}{ }^{\prime}}+\frac{a}{s_{1}{ }^{\prime}-i \omega}+\frac{b}{s_{1}{ }^{\prime}+i \omega}\right) e^{s_{1}{ }^{\prime} t}
\end{aligned}
$$

where

$$
\begin{aligned}
& \alpha_{1}^{\prime}=\frac{1}{\sqrt{2}}\left[\left\{\frac{M^{4}}{\left(1+m^{2}\right)^{2}}+\omega^{2}\right\}^{1 / 2}+\frac{M^{2}}{1+m^{2}}\right]^{1 / 2}, \\
& \beta_{1}^{\prime}=\frac{1}{\sqrt{2}}\left[\left\{\frac{M^{4}}{\left(1+m^{2}\right)^{2}}+\omega^{2}\right\}^{1 / 2}-\frac{M^{2}}{1+m^{2}}\right]^{1 / 2}, \\
& \alpha_{2}^{\prime}=\frac{1}{\sqrt{2}}\left[\left\{\frac{M^{4}}{\left(1+m^{2}\right)^{2}}+4 \omega^{2}\right\}^{1 / 2}+\frac{M^{2}}{1+m^{2}}\right]^{1 / 2},
\end{aligned}
$$




$$
\begin{aligned}
& \beta_{2}{ }^{\prime}=\frac{1}{\sqrt{2}}\left[\left\{\frac{M^{4}}{\left(1+m^{2}\right)^{2}}+4 \omega^{2}\right\}^{1 / 2}-\frac{M^{2}}{1+m^{2}}\right]^{1 / 2}, \\
& \alpha_{3}{ }^{\prime}=\frac{M}{\sqrt{\left(1+m^{2}\right)}}, \\
& \text { and } s_{1}{ }^{\prime}=-\left[n^{2} \pi^{2}+\frac{M^{2}}{1+m^{2}}+i \omega\right] .
\end{aligned}
$$

Equations (24)-(27) represent the solution for fluid velocity in general case. The solutions, Eq. (24) and Eq. (26) exhibit a unified representation of initial MHD Couette flow induced due to non-torsional oscillations of the upper plate, final steady state flow and decaying oscillations excited by interaction of magnetic field, Coriolis force, Hall current and initial oscillatory motion. In the absence of magnetic field (i.e. $M^{2}=0$ ) and Hall current (i.e. $m=0$ ) solutions, Eq. (24) and Eq. (26) are in agreement with the solutions obtained by Das et al. (2008).

We shall now examine the solutions, Eq. (24) and Eq. (26) for large values of time $t$ and for small as well large values of rotation parameter $K^{2}$ and magnetic parameter $M^{2}$.

It may be noted that when time $t$ is large i.e. $\mathrm{t} \gg>1$ then $\omega$ is small (i.e. $\omega<<1$ ) such that $\omega t$ is finite. For large time $t$ and $a=b=\varepsilon / 2$ the solutions, Eq. (24) and Eq. (26) assume the following form

$$
\begin{aligned}
& q(\eta, t) \\
& =\left[1-\frac{\sinh \left(\alpha_{1}+i \beta_{1}\right)(1-\eta)}{\sinh \left(\alpha_{1}+i \beta_{1}\right)}\right] \\
& +\frac{\varepsilon}{2}\left[\left\{1-\frac{\sinh \left(\alpha_{2}+i \beta_{2}\right)(1-\eta)}{\sinh \left(\alpha_{2}+i \beta_{2}\right)}\right\} e^{i \omega t}\right. \\
& \left.+\left\{1-\frac{\sinh \left(\alpha_{3} \pm i \beta_{3}\right)(1-\eta)}{\sinh \left(\alpha_{3} \pm i \beta_{3}\right)}\right\} e^{-i \omega t}\right]
\end{aligned}
$$

for $\omega \neq N^{2}$

$$
\begin{aligned}
& q(\eta, t) \\
& =\left[1-\frac{\sinh \left(\alpha_{1}^{\prime}+i \beta_{1}^{\prime}\right)(1-\eta)}{\sinh \left(\alpha_{1}^{\prime}+i \beta_{1}^{\prime}\right)}\right] \\
& +\frac{\varepsilon}{2}\left[\left\{1-\frac{\sinh \left(\alpha_{2}^{\prime}+i \beta_{2}{ }^{\prime}\right)(1-\eta)}{\sinh \left(\alpha_{2}{ }^{\prime}+i \beta_{2}{ }^{\prime}\right)}\right\} e^{i \omega t}\right. \\
& \left.+\left\{1-\frac{\sinh \alpha_{3}{ }^{\prime}(1-\eta)}{\sinh \alpha_{3}{ }^{\prime}}\right\} e^{-i \omega t}\right]
\end{aligned}
$$

for $\omega=N^{2}$

The solutions, Eq. (28) and Eq. (29) represent the solution for fully developed oscillatory hydromagnetic Couette flow in a rotating system. In the absence of the Hall current (i.e. $m=0$ ) solutions, Eq. (28) and Eq. (29) are in agreement with the solutions obtained by Singh (2000).

\section{AsYmptotic Solution}

We shall now examine the asymptotic behavior of the solution (28) for small as well as large values of $K^{2}$ and $M^{2}$ when $\varepsilon=1$ to gain some physical insight into the flow pattern.

Case I: $\mathrm{K}^{2} \ll<1$ and $\mathrm{M}^{2} \ll<1$

Since $M^{2}, K^{2}$ and $\omega$ are very small, neglecting square and higher powers of $M^{2}, K^{2}$ and $\omega$ in Eq. (28), primary velocity $u_{1}$ and secondary velocity $v_{1}$ assume the following form

$$
\begin{aligned}
u_{1}= & 1+\cos \omega t-(1-\eta)[1+\cos \omega t \\
& +\frac{1}{6}\left(\eta^{2}-2 \eta\right) \\
& \left.\times\left\{\frac{M^{2}}{1+m^{2}}(1+\cos \omega t)-\omega \sin \omega t\right\}\right]+\cdots \\
v_{1}= & -\frac{N^{2}}{6}(1-\eta)\left(\eta^{2}-2 \eta\right)(1+\cos \omega t)+\cdots
\end{aligned}
$$

It is evident from the Eq. (30) and Eq. (31) that for a slowly rotating system with small frequency of oscillations when the conductivity of the fluid is low and/or the applied magnetic field is weak, primary velocity $u_{1}$ is independent of rotation while the secondary velocity $v_{1}$ is affected by magnetic field, Hall current and rotation. The fluid flow in both the directions has considerable effects of oscillations. In the absence of Hall current secondary velocity $v_{1}$ is unaffected by magnetic field.

Case II: $\mathrm{K}^{2}>>1$ and $\mathrm{M}^{2} \sim \mathrm{O}(1)$

When $K^{2}$ is very large and $M^{2}$ is small order of magnitude fluid flow becomes boundary layer type. For the boundary layer flow near the lower plate $\eta=0$, primary velocity $u_{1}$ and secondary velocity $v_{1}$ in Eq. (28) assume the following form

$$
\begin{aligned}
u_{1}= & 1+\cos \omega t-e^{-\alpha_{4} \eta} \cos \beta_{4} \eta \\
& -\frac{1}{2}\left[e^{-\alpha_{5} \eta} \cos \left(\omega t-\beta_{5} \eta\right)\right. \\
& \left.+e^{-\alpha_{6} \eta} \cos \left(\omega t+\beta_{6} \eta\right)\right] \\
v_{1}= & e^{-\alpha_{4} \eta} \sin \beta_{4} \eta-\frac{1}{2}\left[e^{-\alpha_{5} \eta} \sin \left(\omega t-\beta_{5} \eta\right)\right. \\
& \left.-e^{-\alpha_{6} \eta} \sin \left(\omega t \pm \beta_{6} \eta\right)\right]
\end{aligned}
$$

where

$$
\alpha_{4}=K\left[1+\frac{(m+1) M^{2}}{4\left(1+m^{2}\right) K^{2}}\right],
$$




$$
\begin{aligned}
& \beta_{4}=K\left[1+\frac{(m-1) M^{2}}{4\left(1+m^{2}\right) K^{2}}\right], \\
& \alpha_{5}=K\left[1+\frac{\omega}{4 K^{2}}+\frac{(m+1) M^{2}}{4\left(1+m^{2}\right) K^{2}}\right], \\
& \beta_{5}=K\left[1+\frac{\omega}{4 K^{2}}+\frac{(m-1) M^{2}}{4\left(1+m^{2}\right) K^{2}}\right], \\
& \alpha_{6}=K\left[1-\frac{\omega}{4 K^{2}}+\frac{(m+1) M^{2}}{4\left(1+m^{2}\right) K^{2}}\right], \\
& \beta_{6}=K\left[1-\frac{\omega}{4 K^{2}}+\frac{(m-1) M^{2}}{4\left(1+m^{2}\right) K^{2}}\right]
\end{aligned}
$$

It is revealed from the expressions Eq. (32) and Eq. (33) that the solution is in quasi-steady state. Steady state flow is confined within a boundary layer of thickness $O\left(1 / \alpha_{4}\right)$ which may be identified as modified Ekman boundary layer and can be viewed as classical Ekaman boundary layer modified by Hall current and magnetic field. It is noticed from the expressions Eq. (32) and Eq. (33) that the unsteady flow has three modes of oscillations. The first mode corresponds to the pure oscillations of frequency $\omega$ due to non-torsional oscillations of upper plate of the channel which persist in the entire fluid region. The other two modes of oscillations correspond to modified Stokes flow and are confined within boundary layers of thickness $O\left(1 / \alpha_{5}\right)$ and $O\left(1 / \alpha_{6}\right)$. These boundary layers may be recognized as modified Stokes-Ekaman boundary layers and may be viewed as classical Ekman bounadry layers modified by magnetic field, oscillations and Hall current. It may be noted from Eq. (34) that $\alpha_{4}$-layer is thicker than $\alpha_{5}$-layer whereas $\alpha_{6}$-layer is thicker than $\alpha_{4}$-layer. The thickness of $\alpha_{4}$-layer decreases with increase in either $K^{2}$ or $M^{2}$ whereas it increases with increase in $m$. The thickness of $\alpha_{5}$-layer decreases with increase in either $K^{2}$ or $M^{2}$ or $\omega$ whereas it increases with increase in $m$. The thickness of $\alpha_{6}$ layer decreases with increase in either $K^{2}$ or $M^{2}$ whereas it increases with increase in either $\omega$ or $m$. Similar type of boundary layers arise in the neighborhood of the upper plate. It is evident from Eq. (32) and Eq. (33) that unsteady flow is divided into two parts. One part oscillates with amplitude $\frac{1}{2} e^{-\alpha_{5} \eta}$ and the other one with $\frac{1}{2} e^{-\alpha_{6} \eta}$. The unsteady flow corresponding to the former part oscillates with phase lag of $\beta_{5} \eta$ whereas the unsteady flow corresponding to latter part oscillates with a phase lead of $\beta_{6} \eta$.

The exponential terms in Eq. (32) and Eq. (33) damped out quickly as $\eta$ increases. When $\eta \geq 1 / \alpha_{6}$ i.e. outside the boundary layer region, Eq. (32) and Eq. (33) reduce to

$$
u_{1} \approx 1+\cos \omega t, v_{1} \approx 0
$$

It is evident from the expression Eq. (35) that, in a certain core given by $\eta \geq 1 / \alpha_{6}$ i.e. outside the boundary layer region, the fluid has velocity in primary flow direction which oscillates with the same frequency $\omega$ as that of the upper plate when $t>0$.

Case III: $\mathrm{M}^{2}>>1$ and $\mathrm{K}^{2} \sim \mathrm{O}(1)$

This case also corresponds to the boundary layer type flow. For the boundary layer flow near the lower plate $\eta=0$, we obtain fluid velocity from Eq. (28) as

$$
\begin{aligned}
u_{1}= & 1+\cos \omega t-e^{-\alpha_{i} \eta}\left[\cos \beta_{7} \eta\right. \\
& \left.+\frac{1}{2}\left\{\cos \left(\omega t-\beta_{8} \eta\right)+\cos \left(\omega t \pm \beta_{9} \eta\right)\right\}\right] \\
v_{1}= & e^{-\alpha_{i} \eta}\left[\sin \beta_{7} \eta\right. \\
& \left.-\frac{1}{2}\left\{\sin \left(\omega t-\beta_{8} \eta\right)-\sin \left(\omega t \pm \beta_{9} \eta\right)\right\}\right]
\end{aligned}
$$

where

$\alpha_{i}=M / \sqrt{\left(1+m^{2}\right)}$ for $i=7,8,9$,

$\beta_{7}=\frac{N^{2}}{2 M / \sqrt{\left(1+m^{2}\right)}}$,

$\beta_{8}=\frac{\left(\omega+N^{2}\right)}{2 M / \sqrt{\left(1+m^{2}\right)}}$,

$$
\beta_{9}=\frac{\left(\omega-N^{2}\right)}{2 M / \sqrt{\left(1+m^{2}\right)}} \text {. }
$$

The expressions Eq. (36) and Eq. (37) show that the fluid flow is in quasi-steady state. Steady state flow is confined within a modified Hartmann boundary layer of thickness $O\left(1 / \sqrt{M^{2} /\left(1+m^{2}\right)}\right)$ which decreases with increase in magnetic parameter $M^{2}$ and increases with increase in Hall current parameter $m$. Unsteady state flow has three modes of oscillations. The first mode corresponds to pure oscillations with frequency $\omega$ due to non-torsional oscillations of the upper plate of the channel when $t>0$ and fills the entire fluid region. The other two modes of oscillations correspond to the modified Stokes flow and are confined within a thin modified Hartmann boundary layer of thickness $O\left(1 / \sqrt{M^{2} /\left(1+m^{2}\right)}\right)$. Similar type of boundary layer appears adjacent to the upper plate of the channel. It is interesting to note that unsteady flow is divided into two parts and both the parts oscillate with amplitude $\frac{1}{2} e^{-\left(M / \sqrt{1+m^{2}}\right) \eta}$. The unsteady flow corresponding to first part oscillates with phase lag $\beta_{8} \eta$ when $\omega \neq N^{2}$. However, unsteady flow corresponding to second part oscillates with phase lead of $\beta_{9} \eta$ when $\omega<N^{2}$ and this part oscillates with phase lag of $\beta_{9} \eta$ when 
$\omega>N^{2}$. Outside the boundary layer region, the fluid velocity assumes the form

$u_{1} \approx 1+\cos \omega t, v_{1} \approx 0$

It is revealed from Eq. (39) that fluid flows in primary flow direction only and oscillates with the same frequency $\omega$ as that of the upper plate when $t>0$.

\section{Shear Stress at the Plates}

The Non-dimensional shear stress at the lower and upper plates due to primary and secondary flows, for $\omega \neq N^{2}$, are given by

$$
\begin{aligned}
& \tau_{x}+\left.i \tau_{y}\right|_{\eta=0} \\
& =\left(\alpha_{1}+i \beta_{1}\right) \operatorname{coth}\left(\alpha_{1}+i \beta_{1}\right) \\
& +a\left(\alpha_{2}+i \beta_{2}\right) e^{i \omega t} \operatorname{coth}\left(\alpha_{2}+i \beta_{2}\right) \\
& +b\left(\alpha_{3} \pm i \beta_{3}\right) e^{-i \omega t} \operatorname{coth}\left(\alpha_{3} \pm i \beta_{3}\right) \\
& -\sum_{n=1}^{\infty} 2 n^{2} \pi^{2}\left(\frac{1}{s_{1}}+\frac{a}{s_{1}-i \omega}+\frac{b}{s_{1}+i \omega}\right) e^{s_{1} t} \\
& \tau_{x}+\left.i \tau_{y}\right|_{\eta=1} \\
& =\left(\alpha_{1}+i \beta_{1}\right) \operatorname{cosec} \mathrm{h}\left(\alpha_{1}+i \beta_{1}\right) \\
& +a\left(\alpha_{2}+i \beta_{2}\right) e^{i \omega t} \operatorname{cosec} \mathrm{h}\left(\alpha_{2}+i \beta_{2}\right) \\
& +b\left(\alpha_{3} \pm i \beta_{3}\right) e^{-i \omega t} \operatorname{cosec} \mathrm{h}\left(\alpha_{3} \pm i \beta_{3}\right) \\
& -\sum_{n=1}^{\infty}(-1)^{n} 2 n^{2} \pi^{2}\left(\frac{1}{s_{1}}+\frac{a}{s_{1}-i \omega}+\frac{b}{s_{1}+i \omega}\right) e^{s_{1} t}
\end{aligned}
$$

Non-dimensional shear stress at the lower and upper plates due to primary and secondary flows, for $\omega=N^{2}$, are given by

$$
\begin{aligned}
& \tau_{x}+\left.i \tau_{y}\right|_{\eta=0} \\
& =\left(\alpha_{1}{ }^{\prime}+i \beta_{1}{ }^{\prime}\right) \operatorname{coth}\left(\alpha_{1}{ }^{\prime}+i \beta_{1}{ }^{\prime}\right) \\
& +a\left(\alpha_{2}{ }^{\prime}+i \beta_{2}{ }^{\prime}\right) e^{i \omega t} \operatorname{coth}\left(\alpha_{2}{ }^{\prime}+i \beta_{2}{ }^{\prime}\right) \\
& +b \alpha_{3} e^{-i \omega t} \operatorname{coth} \alpha_{3}{ }^{\prime} \\
& -\sum_{n=1}^{\infty} 2 n^{2} \pi^{2}\left(\frac{1}{s_{1}{ }^{\prime}}+\frac{a}{s_{1}{ }^{\prime}-i \omega}+\frac{b}{s_{1}{ }^{\prime}+i \omega}\right) e^{s_{1}{ }^{\prime} t}
\end{aligned}
$$

$$
\begin{aligned}
& \tau_{x}+\left.i \tau_{y}\right|_{\eta=1} \\
& =\left(\alpha_{1}{ }^{\prime}+i \beta_{1}{ }^{\prime}\right) \operatorname{cosec} \mathrm{h}\left(\alpha_{1}{ }^{\prime}+i \beta_{1}{ }^{\prime}\right) \\
& +a\left(\alpha_{2}{ }^{\prime}+i \beta_{2}{ }^{\prime}\right) e^{i \omega t} \operatorname{cosec} \mathrm{h}\left(\alpha_{2}{ }^{\prime}+i \beta_{2}{ }^{\prime}\right) \\
& +b \alpha_{3} e^{-i \omega t} \operatorname{cosec} \mathrm{h} \alpha_{3}{ }^{\prime} \\
& -\sum_{n=1}^{\infty}\left\{(-1)^{n} 2 n^{2} \pi^{2}\right.
\end{aligned}
$$

$\left.\times\left(\frac{1}{s_{1}{ }^{\prime}}+\frac{a}{s_{1}{ }^{\prime}-i \omega}+\frac{b}{s_{1}{ }^{\prime}+i \omega}\right)\right\} e^{s_{1}{ }^{\prime} t}$

\section{RESULTS AND DISCUSSION}

To study the effects of Hall current, rotation, magnetic field and oscillations on the flow-field the numerical values of the fluid velocity, computed from the analytical solution Eq. (24) mentioned in section 2, are displayed graphically versus channel width variable $\eta$ in Figs. 2 to 9 for various values of Hall current parameter $m$, rotation parameter $K^{2}$, magnetic parameter $M^{2}$ and frequency parameter $\omega$ when $\omega t=\pi / 2$ and $\omega \neq N^{2}$. It is evident from Fig. 2 and Fig. 3 that primary velocity $u_{1}$ decreases whereas secondary velocity $v_{1}$ increases on increasing $m$ which implies that Hall current tends to retard primary flow whereas it has reverse effect on secondary flow.

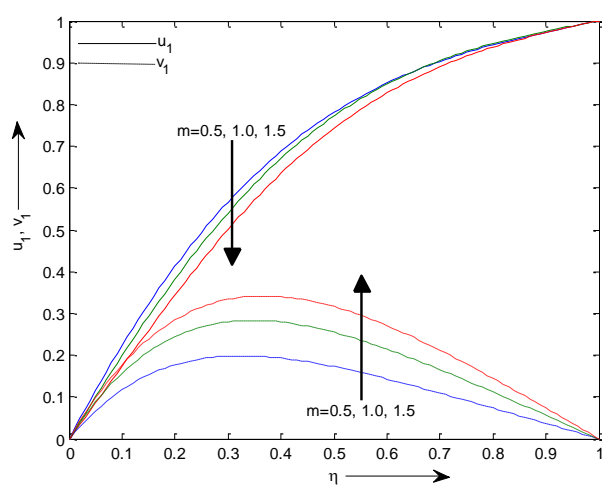

Fig. 2. Velocity profiles when $K^{2}=2$,

$$
M^{2}=10 \text { and } \omega=5\left(\omega<N^{2}\right)
$$

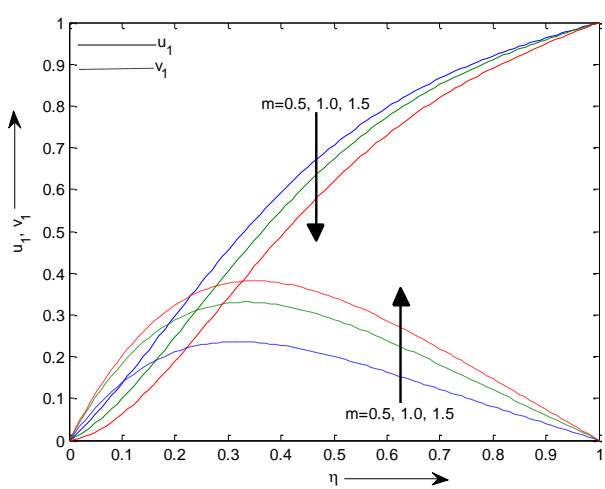

Fig. 3. Velocity profiles when $K^{2}=2$,

$$
M^{2}=10 \text { and } \omega=15\left(\omega>N^{2}\right)
$$


It is revealed from Fig. 4 and Fig. 5 that primary velocity $u_{1}$ and secondary velocity $v_{1}$ increase on increasing $K^{2}$ which implies that rotation tends to accelerate both the primary and secondary flows.

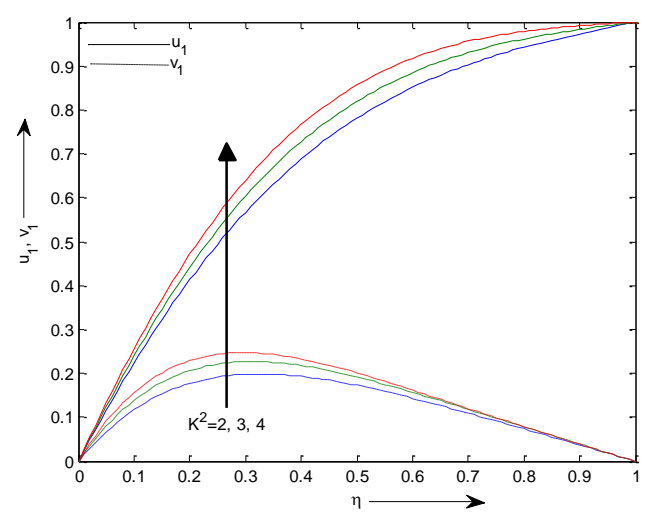

Fig. 4. Velocity profiles when $m=0.5$, $M^{2}=10$ and $\omega=5\left(\omega<N^{2}\right)$

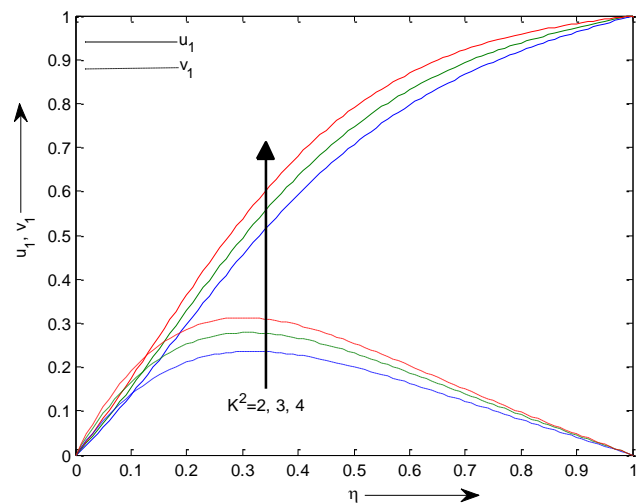

Fig. 5. Velocity profiles when $m=0.5$,

$$
M^{2}=10 \text { and } \omega=15\left(\omega>N^{2}\right)
$$

It is noticed from Fig. 6 and Fig. 7 that primary velocity $u_{1}$ increases whereas secondary velocity $v_{1}$ decreases on increasing $M^{2}$ which implies that magnetic field tends to accelerate primary flow whereas it has reverse effect on secondary flow.

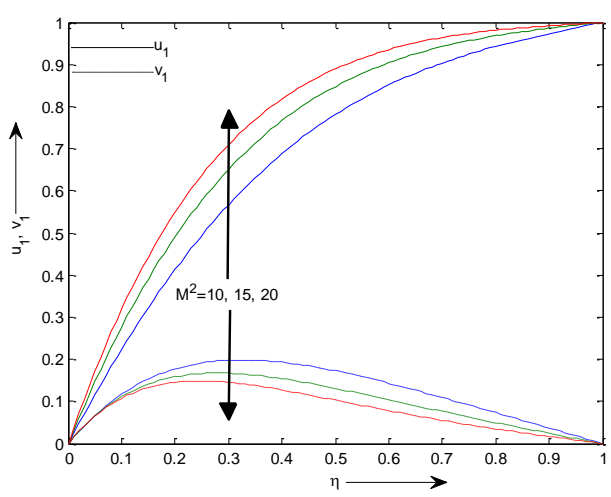

Fig. 6. Velocity profiles when $m=0.5$,

$$
K^{2}=2 \text { and } \omega=5\left(\omega<N^{2}\right)
$$

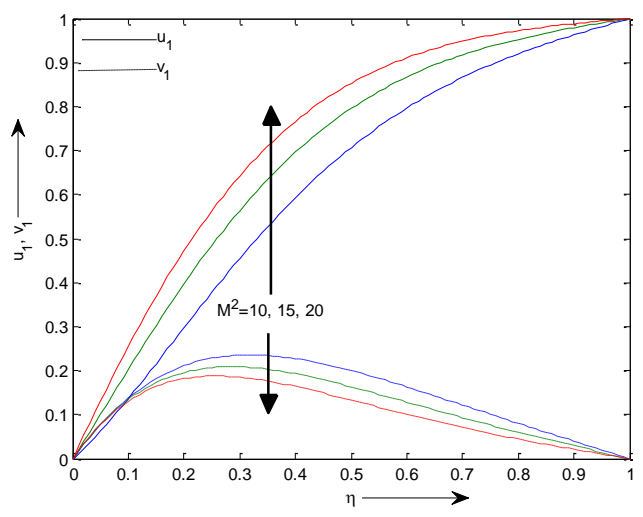

Fig. 7. Velocity profiles when $m=0.5$,

$$
K^{2}=2 \text { and } \omega=15\left(\omega>N^{2}\right)
$$

It is revealed from Fig. 8 and Fig. 9 that primary velocity $u_{1}$ decreases on increasing $\omega$ when $\omega \neq N^{2}$ whereas the secondary velocity $v_{1}$ increases on increasing $\omega$ when $\omega<N^{2}$ and it decreases on increasing $\omega$ when $\omega>N^{2}$ which implies that oscillations tend to retard primary flow when $\omega \neq N^{2}$ and secondary flow when $\omega>N^{2}$ whereas it have reverse effect on secondary flow when $\omega<N^{2}$.

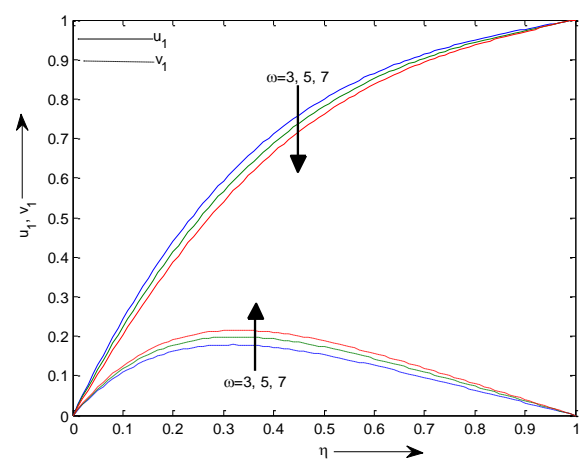

Fig. 8. Velocity profiles when $m=0.5$,

$$
K^{2}=2 \text { and } M^{2}=10\left(\omega<N^{2}\right)
$$

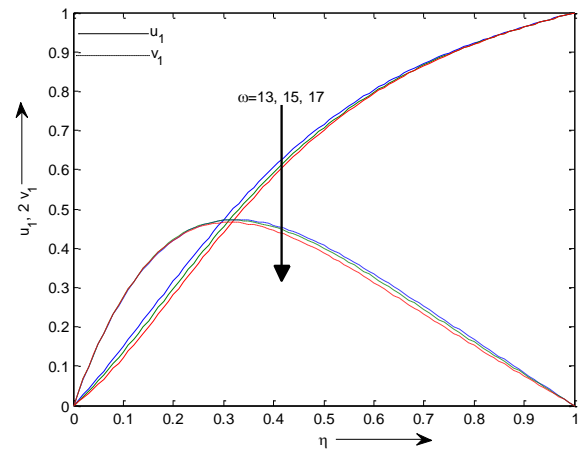

Fig. 9. Velocity profiles when $m=0.5$,

$$
K^{2}=2 \text { and } M^{2}=10\left(\omega>N^{2}\right)
$$


The numerical values of the primary and secondary shear stress at the lower and upper plates, computed from the analytical expressions Eq. (40) and Eq. (41) mentioned in section 4 , are presented in tabular form in Tables 1-8 for various values of $m, \omega, K^{2}$ and $M^{2}$ when $\omega \neq N^{2}$ by considering $\omega t=\pi / 2$. It is evident from Table 1 that, for $\omega<N^{2}$, primary shear stress at the lower plate i.e. $\left.\tau_{x}\right|_{\eta=0}$ decreases whereas secondary shear stress at the lower plate i.e. $\left.\tau_{y}\right|_{\eta=0}$ increases on increasing either $m$ or $\omega$. This implies that Hall current and oscillations tend to reduce primary shear stress at the lower plate whereas it have reverse effect on secondary shear stress at the lower plate when $\omega<N^{2}$.

Table 1 Shear stress at the lower plate due to primary and secondary flows when $K^{2}=2$ and $M^{2}=10\left(\omega<N^{2}\right)$

\begin{tabular}{|c|c|c|c|c|}
\cline { 2 - 5 } \multicolumn{2}{c|}{$\omega \downarrow m \rightarrow$} & 0.5 & 1.0 & 1.5 \\
\hline \multirow{3}{*}{$\left.\tau_{x}\right|_{\eta=0}$} & 3 & 2.6795 & 2.3162 & 1.9845 \\
\cline { 2 - 5 } & 5 & 2.3999 & 2.0254 & 1.6727 \\
\hline \multirow{3}{*}{$\left.\tau_{y}\right|_{\eta=0}$} & 7 & 2.1227 & 1.7286 & 1.3479 \\
\cline { 2 - 5 } & 3 & 1.469 & 1.849 & 2.0177 \\
\cline { 2 - 5 } & 5 & 1.5332 & 1.9886 & 2.1874 \\
\hline
\end{tabular}

It is observed from Table 2 that, for $\omega>N^{2}$, on increasing $m,\left.\quad \tau_{x}\right|_{\eta=0} \quad$ decreases when $\omega=13$ and 15 and it decreases, attains a minimum and then increases in magnitude when $\omega=17$ whereas, on increasing $\omega,\left.\tau_{x}\right|_{\eta=0}$ decreases when $m=0.5$ and 1.0 and it decreases, attains a minimum and then increases in magnitude when $m=1.5$. $\left.\tau_{y}\right|_{\eta=0}$ increases on increasing $m$. On increasing $\omega$, $\left.\tau_{y}\right|_{\eta=0}$ increases when $m=0.5$, it increases, attains a maximum and then decreases when $m=1.0$ and it decreases when $m=1.5$. This implies that, for $\omega>N^{2}$, Hall current tends to enhance secondary shear stress at the lower plate whereas it has reverse effect on the primary shear stress at the lower plate when $\omega \leq 15$. Oscillations tend to reduce primary shear stress at the lower plate when $m \leq 1.0$ and secondary shear stress at the lower plate behaves in oscillatory manner with respect to oscillations. It may be noted from Table 2 that there exists flow separation at the lower plate in the primary flow direction on increasing either $m$ or $\omega$. It is revealed from Table 3 that, for $\omega<N^{2}$, primary shear stress at the upper plate i.e. $\left.\tau_{x}\right|_{\eta=1}$ and secondary shear stress at the upper plate i.e. $\left.\tau_{y}\right|_{\eta=1}$ increase on increasing $\omega$. $\left.\tau_{x}\right|_{\eta=1}$ decreases, attains a minimum and then increases on increasing $\left.m \cdot \tau_{y}\right|_{\eta=1}$ increases on increasing $m$. This implies that, for $\omega<N^{2}$, Hall current tends to enhance secondary shear stress at the upper plate whereas oscillations have tendency to enhance both the primary and secondary shear stress at the upper plate.

Table 2 Shear stress at the lower plate due to primary and secondary flows when $K^{2}=2$ and $M^{2}=10\left(\omega>N^{2}\right)$

\begin{tabular}{|c|c|c|c|c|}
\cline { 2 - 5 } \multicolumn{2}{c|}{$\omega \downarrow m \rightarrow$} & 0.5 & 1.0 & 1.5 \\
\hline \multirow{3}{*}{$\tau_{x} \mid \eta=0$} & 13 & 1.3378 & 0.8603 & 0.4007 \\
\cline { 2 - 5 } & 15 & 1.1021 & 0.6027 & 0.1335 \\
\cline { 2 - 5 } & 17 & 0.8818 & 0.3674 & -0.1031 \\
\hline \multirow{3}{*}{$\left.\tau_{y}\right|_{\eta=0}$} & 13 & 1.7505 & 2.2758 & 2.4749 \\
\cline { 2 - 5 } & 15 & 1.7643 & 2.2803 & 2.4585 \\
\cline { 2 - 5 } & 17 & 1.7680 & 2.2701 & 2.4288 \\
\hline
\end{tabular}

Table 3 Shear stress at the upper plate due to primary and secondary flows when $K^{2}=2$ and $M^{2}=10\left(\omega<N^{2}\right)$

\begin{tabular}{|c|c|c|c|c|}
\cline { 2 - 5 } \multicolumn{2}{c|}{$\omega \downarrow m \rightarrow$} & 0.5 & 1.0 & 1.5 \\
\hline \multirow{3}{*}{$\left.\tau_{x}\right|_{\eta=1}$} & 3 & 0.2296 & 0.2100 & 0.2426 \\
\cline { 2 - 5 } & 5 & 0.2588 & 0.2360 & 0.2755 \\
\cline { 2 - 5 } & 7 & 0.2885 & 0.2712 & 0.3251 \\
\hline \multirow{3}{*}{$-\left.\tau_{y}\right|_{\eta=1}$} & 3 & 0.3197 & 0.4907 & 0.6374 \\
\cline { 2 - 5 } & 5 & 0.3672 & 0.5661 & 0.7381 \\
\cline { 2 - 5 } & 7 & 0.4018 & 0.6219 & 0.8094 \\
\hline
\end{tabular}

It is noticed from Table 4 that, for $\omega>N^{2},\left.\tau_{x}\right|_{\eta=1}$ and $\left.\tau_{y}\right|_{\eta=1}$ increase on increasing $m .\left.\tau_{x}\right|_{\eta=1}$ increases when $m=0.5$ and 1.0 and it increases, attains a maximum and then decreases when $m=1.5$ on increasing $\omega .\left.\tau_{y}\right|_{\eta=1}$ decreases on increasing $\omega$. This implies that, for $\omega>N^{2}$, Hall current tends to enhance both the primary and secondary shear stress at the upper plate and oscillations tends to reduce secondary shear stress at the upper plate while it tends to enhance primary shear stress at the upper plate when $m \leq 1.0$. It is observed from Table 5 that, for $\omega<N^{2},\left.\tau_{x}\right|_{\eta=0}$ and $\left.\tau_{y}\right|_{\eta=0}$ increase on increasing $K^{2}$. On increasing $M^{2},\left.\tau_{x}\right|_{\eta=0}$ decreases when $K^{2}=2$ and it increases when $K^{2}=3$ and 4 . On increasing $M^{2},\left.\tau_{y}\right|_{\eta=0}$ increases when $K^{2}=2$, it decreases, attains a minimum and then increases when $K^{2}=3$ and it decreases when $K^{2}=4$. This implies 
that, for $\omega<N^{2}$, rotation tends to enhance both the primary and secondary shear stress at the lower plate. Magnetic field tends to enhance primary shear stress at the lower plate when $K^{2} \geq 3$. Secondary shear stress at the lower plate behaves in oscillatory manner with respect to magnetic field.

Table 4 Shear stress at the upper plate due to primary and secondary flows when $K^{2}=2$ and $M^{2}=10\left(\omega>N^{2}\right)$

\begin{tabular}{|c|c|c|c|c|}
\cline { 3 - 5 } \multicolumn{2}{c|}{$\omega \downarrow m \rightarrow$} & 0.5 & 1.0 & 1.5 \\
\hline \multirow{3}{*}{$\left.\tau_{x}\right|_{\eta=1}$} & 13 & 0.3575 & 0.3848 & 0.4826 \\
\cline { 2 - 5 } & 15 & 0.3662 & 0.4041 & 0.5021 \\
\cline { 2 - 5 } & 17 & 0.3665 & 0.4091 & 0.5003 \\
\hline \multirow{3}{*}{$-\left.\tau_{y}\right|_{\eta=1}$} & 13 & 0.4165 & 0.6368 & 0.7964 \\
\cline { 2 - 5 } & 15 & 0.3977 & 0.6007 & 0.7384 \\
\cline { 2 - 5 } & 17 & 0.3731 & 0.5559 & 0.6740 \\
\hline
\end{tabular}

Table 5 Shear stress at the lower plate due to primary and secondary flows when $m=0.5$ and $\omega=5\left(\omega<N^{2}\right)$

\begin{tabular}{|c|c|c|c|c|}
\cline { 3 - 5 } \multicolumn{1}{c|}{$M^{2} \downarrow K^{2} \rightarrow$} & 2 & 3 & 4 \\
\hline \multirow{4}{*}{$\left.\tau_{x}\right|_{\eta=0}$} & 10 & 2.3999 & 2.5680 & 2.7420 \\
\cline { 2 - 5 } & 15 & 3.1140 & 3.2357 & 3.3629 \\
\cline { 2 - 5 } & 20 & 3.7094 & 3.8050 & 3.9054 \\
\hline \multirow{3}{*}{$\left.\tau_{y}\right|_{\eta=0}$} & 10 & 1.5332 & 1.8322 & 2.0963 \\
\cline { 2 - 5 } & 15 & 1.5468 & 1.8002 & 2.0337 \\
\cline { 2 - 5 } & 20 & 1.5848 & 1.8083 & 2.0190 \\
\hline
\end{tabular}

It is evident from Table 6 that, for $\omega>N^{2},\left.\tau_{x}\right|_{\eta=0}$ and $\left.\tau_{y}\right|_{\eta=0}$ increase on increasing $K^{2} .\left.\tau_{x}\right|_{\eta=0}$ increases on increasing $\left.M^{2} \cdot \tau_{y}\right|_{\eta=0}$ increases when $K^{2}=2$ and it decreases when $K^{2}=3$ and 4 on increasing $M^{2}$. This implies that, for $\omega>N^{2}$, rotation tends to enhance both the primary and secondary shear stress at the lower plate. Magnetic field tends to enhance primary shear stress at the lower plate whereas it has reverse effect on the secondary shear stress at the lower plate when $K^{2} \geq 3$. It is observed from Table 7 that, for $\omega<N^{2},\left.\quad \tau_{x}\right|_{\eta=1}$ decreases whereas $\left.\tau_{y}\right|_{\eta=1}$ increases, attains a maximum and then increases on increasing $\left.K^{2} \cdot \tau_{x}\right|_{\eta=1}$ and $\left.\tau_{y}\right|_{\eta=1}$ decreases on increasing $M^{2}$. This implies that, for $\omega<N^{2}$, rotation tends to reduce primary shear stress at the upper plate. Magnetic field tends to reduce both the primary and secondary shear stress at the upper plate.
Table 6 Shear stress at the lower plate due to primary and secondary flows when $m=0.5$ and $\omega=15\left(\omega>N^{2}\right)$

\begin{tabular}{|c|c|c|c|c|}
\cline { 2 - 5 } \multicolumn{1}{c|}{$M^{2} \downarrow K^{2} \rightarrow$} & 2 & 3 & 4 \\
\hline \multirow{4}{*}{$\left.\tau_{x}\right|_{\eta=0}$} & 10 & 1.1021 & 1.2890 & 1.4972 \\
\cline { 2 - 5 } & 15 & 1.9593 & 2.1080 & 2.2695 \\
\cline { 2 - 5 } & 20 & 2.6682 & 2.7893 & 2.9189 \\
\hline \multirow{3}{*}{$\left.\tau_{y}\right|_{\eta=0}$} & 10 & 1.7643 & 2.1373 & 2.4744 \\
\cline { 2 - 5 } & 15 & 1.8020 & 2.1065 & 2.3867 \\
\cline { 2 - 5 } & 20 & 1.8372 & 2.0972 & 2.3405 \\
\hline
\end{tabular}

Table 7 Shear stress at the upper plate due to primary and secondary flows when $m=0.5$

\begin{tabular}{|c|c|c|c|c|}
\multicolumn{6}{c}{ and $\omega=5\left(\omega<N^{2}\right)$} \\
\cline { 2 - 5 } \multicolumn{1}{c}{$M^{2} \downarrow K^{2} \rightarrow$} & 2 & 3 & 4 \\
\hline \multirow{3}{*}{$\left.\tau_{x}\right|_{\eta=1}$} & 10 & 0.2588 & 0.1618 & 0.0718 \\
\cline { 2 - 5 } & 15 & 0.1365 & 0.0820 & 0.0316 \\
\cline { 2 - 5 } & 20 & 0.0740 & 0.0414 & 0.0112 \\
\hline \multirow{3}{*}{$-\left.\tau_{y}\right|_{\eta=1}$} & 10 & 0.3672 & 0.3894 & 0.3859 \\
\cline { 2 - 5 } & 15 & 0.2431 & 0.2509 & 0.2464 \\
\cline { 2 - 5 } & 20 & 0.1662 & 0.1683 & 0.1640 \\
\hline
\end{tabular}

It is found from Table 8 that, for $\omega>N^{2},\left.\tau_{x}\right|_{\eta=1}$ decreases whereas $\left.\tau_{y}\right|_{\eta=1}$ increases on increasing $\left.K^{2} \cdot \tau_{x}\right|_{\eta=1}$ and $\left.\tau_{y}\right|_{\eta=1}$ decrease on increasing $M^{2}$. This implies that, for $\omega>N^{2}$, rotation tends to reduce primary shear stress at the upper plate whereas it has reverse effect on secondary shear stress at the upper plate. Magnetic field has tendency to reduce both the primary and secondary shear stress at the upper plate.

Table 8 Shear stress at the upper plate due to primary and secondary flows when $m=0.5$ and $\omega=15\left(\omega>N^{2}\right)$

\begin{tabular}{|c|c|c|c|c|}
\cline { 3 - 5 } \multicolumn{1}{c|}{$M^{2} \downarrow K^{2} \rightarrow$} & 2 & 3 & 4 \\
\hline \multirow{4}{*}{$\left.\tau_{x}\right|_{\eta=1}$} & 10 & 0.3662 & 0.2706 & 0.1703 \\
\cline { 2 - 5 } & 15 & 0.2033 & 0.1407 & 0.0777 \\
\cline { 2 - 5 } & 20 & 0.1127 & 0.0718 & 0.0319 \\
\hline \multirow{4}{*}{$-\left.\tau_{y}\right|_{\eta=1}$} & 10 & 0.3977 & 0.4481 & 0.4742 \\
\cline { 2 - 5 } & 15 & 0.2913 & 0.3133 & 0.3210 \\
\cline { 2 - 5 } & 20 & 0.2111 & 0.2198 & 0.2202 \\
\hline
\end{tabular}

\section{CONCLUSION}

The Present investigation deals with the theoretical study of unsteady MHD Couette flow of class-II in a rotating system. The significant results are summarized below: 
1. Hall current tends to retard primary flow whereas it has reverse effect on secondary flow.

2. Rotation tends to accelerate both the primary and secondary flows.

3. Magnetic field tends to accelerate primary flow whereas it has reverse effect on secondary flow.

4. Oscillations tend to retard primary flow when $\omega \neq N^{2}$ and secondary flow when $\omega>N^{2}$ whereas it has reverse effect on secondary flow when $\omega<N^{2}$.

5. For $\omega<N^{2}$, Hall current and oscillations tend to reduce primary shear stress at the lower plate whereas it have reverse effect on secondary shear stress at the lower plate.

6. For $\omega>N^{2}$, Hall current tends to enhance secondary shear stress at the lower plate whereas it has reverse effect on the primary shear stress at the lower plate when $\omega \leq 15$. Oscillations tend to reduce primary shear stress at the lower plate when $m \leq 1.0$ and secondary shear stress at the lower plate behaves in oscillatory manner with respect to oscillations. There exists flow separation at the lower plate in primary flow direction on increasing either $m$ or $\omega$.

7. For $\omega<N^{2}$, Hall current tends to enhance secondary shear stress at the upper plate whereas oscillations have tendency to enhance both the primary and secondary shear stress at the upper plate.

8. For $\omega>N^{2}$, Hall current tends to enhance both the primary and secondary shear stress at the upper plate and oscillations tends to reduce secondary shear stress at the upper plate while it tends to enhance primary shear stress at the upper plate when $m \leq 1.0$.

9. For $\omega<N^{2}$, rotation tends to enhance both the primary and secondary shear stress at the lower plate. Magnetic field tends to enhance primary shear stress at the lower plate when $K^{2} \geq 3$. Secondary shear stress at the lower plate behaves in oscillatory manner with respect to magnetic field.

10. For $\omega>N^{2}$, rotation tends to enhance both the primary and secondary shear stress at the lower plate. Magnetic field tends to enhance primary shear stress at the lower plate whereas it has reverse effect on the secondary shear stress at the lower plate when $K^{2} \geq 3$.

11. For $\omega<N^{2}$, rotation tends to reduce primary shear stress at the upper plate. Magnetic field tends to reduce both the primary and secondary shear stress at the upper plate.

12. For $\omega>N^{2}$, rotation tends to reduce primary shear stress at the upper plate whereas it has reverse effect on secondary shear stress at the upper plate. Magnetic field has tendency to reduce both the primary and secondary shear stress at the upper plate.

\section{ACKNOWLEDGEMENTS}

One of the authors (J. K. Singh) is thankful to CSIR, New Delhi (India) for providing financial assistance to carry out this research work.

\section{REFERENCES}

Beg, O.A., S.K. Ghosh and M. Narahari (2011). Mathematical modeling of oscillatory MHD Couette flow in rotating highly permeable medium permeated by an oblique magnetic field, Chemical Engineering and Communications, 198, 235-254.

Chandran, P., N.C. Sacheti and A.K. Singh (1993). Effect of rotation on unsteady hydromagnetic Couette flow, Astrophys. Space Sci., 202, 1-10.

Cowling, T.G. (1957). Magnetohydrodynamics, Interscience Publishers, New York.

Cramer, K.R. and Pai, S.I. (1973). Magnetofluiddynamics for Engineers and Applied Physicists, McGraw Hill Book Company, New York.

Das, B.K., M. Guria and R.N. Jana (2008). Unsteady Couette flow in a rotating system, Meccanica, 43, 517-521.

Das, S., S.L. Maji, M. Guria and R.N. Jana (2009). Unsteady MHD Couette flow in a rotating system, Mathematical and Computer Modeling, 50, 12111217.

Ghosh, S.K. (2002). Effects of Hall current on MHD Couette flow in a rotating system with arbitrary magnetic field, Czech. J. Phys., 52, 51-63.

Ghosh, S.K. and I. Pop (2004). Hall effects on MHD plasma Couette flow in a rotating environment, Int. J. Appl. Mech. Eng., 9, 293-305.

Guria, M., S. Das, R.N. Jana and S.K. Ghosh (2009). Oscillatory Couette flow in the presence of inclined magnetic field, Meccanica, 44, 555-564.

Guria, M., R.N. Jana and S.K. Ghosh (2008). Effect of wall conductance on MHD Couette flow and heat transfer in a rotating system, Int. J. Appl. Mech. Eng., 13, 75-90.

Hayat, T., S. Nadeem and S. Asghar (2004a). Hydromagnetic Couette flow of a Oldroyd-B fluid in a rotating system, Int. J. Engng. Sci., 42, 65-78.

Hayat, T., S. Nadeem, A.M. Siddiqui and S. Asghar (2004b). An oscillatory hydromagnetic NonNewtonian flow in a rotating system, Appl. Math. Lett., 17, 609-614. 
Hayat, T., Y. Wang and K. Hutter (2004c). Hall effects on the unsteady hydromagnetic oscillatory flow of a Second-Grade fluid, Int. J. Non-linear Mech., 39, (6), 1027-1037.

Katagiri, M. (1962). Flow formation in Couette motion in Magnetohydrodynamics, J. Phy. Soc. Jpn., 17, 393-396.

Meyer, R.C. (1958). On reducing aerodynamic heattransfer rates by Magnetohydrodynamic technique, J. Aero. Sci., 25, 561-572.

Mishra, S.P. and J.C. Muduli (1980). Unsteady flow through two porous flat walls in the presence of a magnetic field, Rev. Roum. des. Sci. Tech. Serie de Mech. Appl., 25, 21-27.

Muhuri, P.K. (1963). Flow formation in Couette motion in Magnetohydrodynamics with suction, J. Phy. Soc. Jpn., 18, 1671-1675.

Seth, G.S., R.N. Jana and M.K. Maiti (1982). Unsteady hydromagnetic Couette flow in a rotating system, Int. J. Engng. Sci., 20, 989-999.

Seth, G.S., R. Singh and N. Mahto (1988). Oscillatory hydromagnetic Couette flow in a rotating system, Ind. J. Tech., 26, 329-333.

Seth, G.S., R. Nandkeolyar and Md. S. Ansari (2009). Hall effects on oscillatory hydromagnetic Couette flow in a rotating system, Int. J. Acad. Res., 1(2), 617.

Seth, G.S., Md. S. Ansari and R. Nandkeolyar (2010a). Unsteady hydromagnetic Couette flow within porous plates in a rotating system, Adv. Appl. Math. Mech., 2(3), 286-302.

Seth, G.S., Md. S. Ansari and R. Nandkeolyar (2010b). Unsteady hydromagnetic Couette flow induced due to accelerated movement of one of the porous plates of the channel in a rotating system, Int. J. Appl. Math. Mech., 6(7), 24-42.
Seth, G.S., Md. S. Ansari and R. Nandkeolyar (2011a). Unsteady hydromagnetic flow within a porous channel, Tamkang J. Sci. and Eng., 14(1), 7-14.

Seth, G.S., Md. S. Ansari and R. Nandkeolyar (2011b). Effects of rotation and magnetic field on unsteady Couette flow in a porous channel, J. Appl. Fluid Mech., 4(2), 95-103.

Seth, G.S., S.M. Hussain and J.K. Singh (2011c). MHD Couette flow of class-II in a rotating system, $J$. Appl. Math. and Bioinformatics, 1(1), 31-54.

Seth, G.S., R. Nandkeolyar and Md. S. Ansari (2012). Effects of Hall current and rotation on unsteady MHD Couette flow in the presence of an inclined magnetic field, J. Appl. Fluid Mech., 5(2), 67-74.

Seth, G.S. and J.K. Singh (2011). Steady hydromagnetic Couette flow in a rotating system with nonconducting walls, Int. J. Eng. Sci. and Tech., 3(2), 146-156.

Singh, A.K. and N. Kumar, (1983). Unsteady Magnetohydrodynamic Couette flow, Wear., 89, 125-129.

Singh, A.K., N.C. Sacheti, and P. Chandran, (1994). Transient effects on Magnetohydrodynamic Couette flow with rotation: Accelerated motion, Int. J. Engng. Sci., 32, 133-139.

Singh, K.D. (2000). An oscillatory hydromagnetic Couette flow in a rotating system, ZAMM, 80, 429432 .

Soundalgekar, V.M. (1967). On the flow of an electrically conducting incompressible fluid near an accelerated plate in the presence of a parallel plate, under transverse magnetic field, Proc. Ind. Acad. Sci., 65A, 179-187.

Tao, L.N. (1960). Magnetohydrodynamic effects on the formation of Couette flow, J. Aerospace Sci., 27, 334-338. 